\title{
Magnetoresistance and Irreversibility Fields of Bismuth-Based 1G Tape
}

\author{
W. M. Woch ${ }^{1}$ - M. Chrobak $^{1} \cdot$ M. Kowalik ${ }^{1} \cdot$ R. Zalecki ${ }^{1} \cdot$ J. Przewoźnik $^{1}$ \\ Cz. Kapusta ${ }^{1}$
}

Received: 17 March 2016 / Accepted: 27 April 2016 / Published online: 14 May 2016

(C) The Author(s) 2016. This article is published with open access at Springerlink.com

\begin{abstract}
The magnetoresistance and irreversibility fields of commercial bismuth-based $1 \mathrm{G}$ tape were studied on the basis of the temperature dependencies of the magnetoresistance at the two relative orientations of magnetic field and superconductor plane. The critical temperatures of this tape are the following: $T_{\mathrm{c} 50 \%}=110.3 \mathrm{~K}$ and $T_{\mathrm{c} 0}=109.9 \mathrm{~K}$, and the width of superconducting transition is $\Delta T=0.5 \mathrm{~K}$. The widths of the transition to the superconducting state versus applied magnetic fields were derived for both orientations. The experimental data were fitted using the formula $\Delta T=C H^{m}+\Delta T_{0}$. The irreversibility field values were obtained and successfully fitted as a function of temperature. At $77 \mathrm{~K}$, they were found to amount to $H_{i r r}=72.8 \mathrm{kOe}$ and $H_{i r}=5.5 \mathrm{kOe}$ for the parallel and perpendicular directions, respectively.
\end{abstract}

Keywords 1G superconducting tape - Width of superconducting transition $\cdot$ Irreversibility fields

\section{Introduction}

The successful preparation of high-temperature superconductor (HTS) films and tapes has opened new opportunities for large-scale applications including power system components such as electrical motors, generators, power transmission cables, transformers, magnets, and

W. M. Woch

wmwoch@agh.edu.pl

1 Faculty of Physics and Applied Computer Science, Department of Solid State Physics, AGH University of Science and Technology, Av. Mickiewicza 30, 30-059 Kraków, Poland superconducting magnetic energy storage devices [1-3]. Extensive investigations have been focused on optimizing the fabrication and processing techniques of HTS wires and tapes [4] giving the high values of critical current densities and irreversibility fields. Typical critical current densities of the first-generation (1G) bismuth-based tapes are of the order of $30-35 \mathrm{kA} \mathrm{cm}^{-2}$ at $77 \mathrm{~K}$ and the self magnetic field that gives the critical currents of the order of 120-170 A [5-7]. These values decrease to 20-60 A in the magnetic field $B=1 \mathrm{~T}$ at $77 \mathrm{~K}$. One of the highest reported critical current densities was about $115 \mathrm{kA} \mathrm{cm}^{-2}$ at $77 \mathrm{~K}[8,9]$. The high-temperature superconductors exhibit special features in comparison to the classical ones. They are extreme second type with a large penetration depth $\lambda$ of the order of $10^{3}-10^{4} \AA$ [10] and very small coherence length $\xi$ of the order of $10 \AA$ [11]. That makes the Ginzburg-Landau parameter $\kappa=\lambda / \xi$ is of the order of $10^{2}$ or more. Other important superconducting parameters are also extremely anisotropic (e.g. the ratio of the $c$-axis to the $a-b$-plane direction of penetration depths $\gamma=\lambda_{\mathrm{c}} / \lambda_{\mathrm{ab}}$ is of the order of $10-10^{2}$ for YBCO and of the order of $10^{4}$ for BSCCO) like the critical currents, the irreversibility fields, and others. This is due to the anisotropic layered structure of weakly coupled $\mathrm{CuO}_{2}$ superconducting planes.

In this paper, the temperature dependencies of the magnetoresistance of commercial bismuth-based $1 \mathrm{G}$ tape were studied and the field dependencies of the transition width as well as temperature dependencies of the irreversibility fields are reported and analysed.

\section{Experiment}

The commercial first-generation bismuth-based superconducting tape used in the experiment was manufactured 
by the American Superconductor Company (AMSC). The commercial identifier of this tape is the following: $1 \mathrm{G}$ High Strength Plus 135A Ic min "5715 135" BSCCO.

The temperature dependencies of the magnetoresistance were measured using the standard four-point a.c. method option of the Quantum Design PPMS apparatus with $90 \mathrm{kOe}$ superconducting magnet. The measurements were carried out for the two relative configurations of the tape plane and the applied magnetic field: perpendicular and parallel.

\section{Results and Discussion}

The temperature dependencies of the magnetoresistance for the perpendicular and parallel magnetic field orientations with respect to the tape plane are shown in Fig. 1a, b, respectively. At the zero field, the critical temperatures for this tape are as follows: $T_{\mathrm{c} 50 \%}=110.3 \mathrm{~K}$ and $T_{\mathrm{c} 0}=109.9 \mathrm{~K}$, and the width of superconducting transition is $\Delta T_{0}=0.5 \mathrm{~K}$. One can notice a significant broadening of the superconducting transition with the application of the external magnetic field, which is typical for HTS [12, 13]. Some authors link this phenomenon with the vortex structure and flux motion mechanism $[14,15]$. On the other hand, the resistive transition from the normal to the superconducting state of
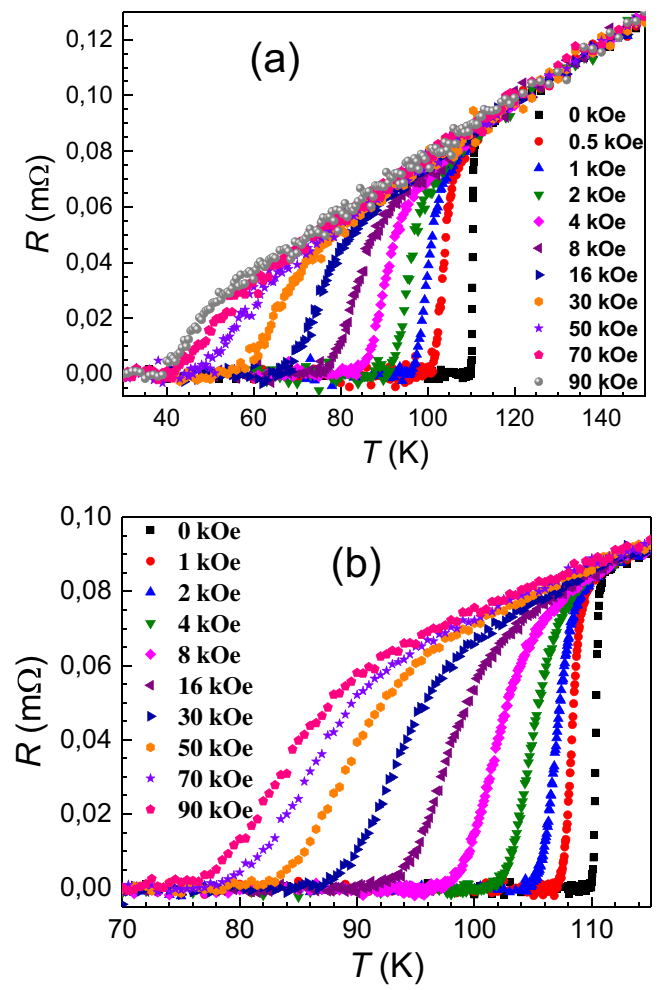

Fig. 1 Temperature dependencies of the magnetoresistance for the perpendicular $\mathbf{a}$ and the parallel $\mathbf{b}$ orientation of the applied magnetic field with respect to the surface of the tape
HTS hides many interesting physical phenomena [16] and is possibly a key to understand the mechanism of a superconductivity of these materials. From this point of view, the attention is paid to the width of the resistive transition that is usually defined as $\Delta T=T_{90} \%-T_{10} \%$ and its dependence on the applied magnetic field. To take advantage of the experimental curves that are shown in Fig. 1, the widths of the transition versus applied magnetic fields were derived for both directions and they are shown in Fig. 2. For HTS, the resistive transition is relatively wide already for $H=0$. The width of the resistive transition can generally be described in the following form [17]:

$\Delta T=C H^{m}+\Delta T_{0}$

where $m=2 / 3$ and $\Delta T_{0}$ means the width at the zero applied magnetic field. The exponent $m$ is related to the exponent $n$ that appears in the temperature dependence of the irreversibility field by the relation: $m=1 / n$. The coefficient $C$ depends on the critical current at zero magnetic field as well as on the critical temperature. The experimental data presented in Fig. 2 (open squares for the plane of the tape perpendicular to the applied magnetic field and open circles for parallel direction) were well fitted using (1) (the solid lines in Fig. 2). For the each direction, the two parameters, $m$ and $C$, were used as the fit parameters in (1). The width of the resistive transition at the zero applied magnetic field $\Delta T_{0}=0.5 \mathrm{~K}$ was taken from the experimental data. The parameters $m$ and $C$ obtained from the fitting procedure are $0.41 \pm 0.02$ and $8.4 \pm 0.7$ for the perpendicular direction and $0.51 \pm 0.03$ and $2.04 \pm 0.24$ for the parallel direction, respectively. The critical exponents derived from these fits are generally smaller than the theoretical $m=2 / 3$ in (1). Smaller $m$ corresponds to a more two-dimensional vortex structure [18], and it means that the pining force is rather strong and, as a consequence, the critical current can

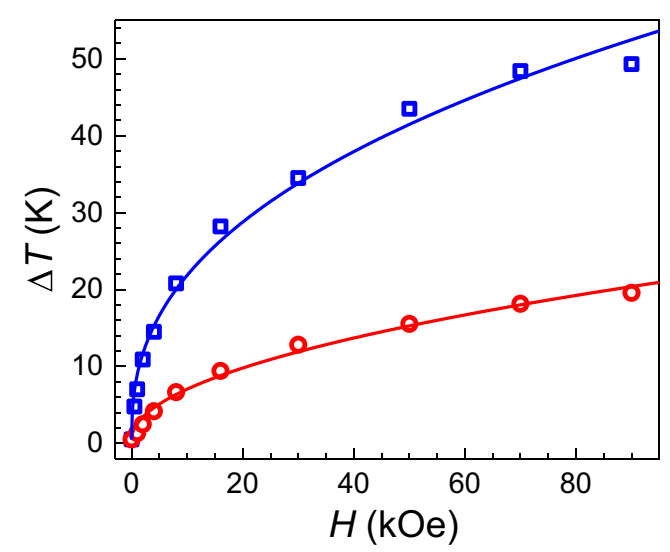

Fig. 2 The width of the transition versus the applied magnetic field for the perpendicular (open squares) and the parallel (open circles) orientation of the applied magnetic field with respect to the surface of the tape. Solid lines are the fits to (1) 
reach higher values. Indeed, the density of the critical current of this tape is $J_{\mathrm{c}}=1.710^{4} \mathrm{~A} / \mathrm{cm}^{-2}$ at $77 \mathrm{~K}$ in the self magnetic field [19]. One can notice that at the zero applied magnetic field the width of the resistive transition is very small and rises when the magnetic field is increased. The width of the resistive transition starts from $\Delta T_{0}=0.5 \mathrm{~K}$ at the zero applied magnetic field and reaches $\Delta T=20.4 \mathrm{~K}$ at $90 \mathrm{kOe}$ for the parallel and $\Delta T=52.5 \mathrm{~K}$ at $90 \mathrm{kOe}$ for the perpendicular orientations. Similarly to the other structural and superconducting parameters, the width of the resistive transition also shows the anisotropy depending on the applied magnetic field.

The most important curve on the H-T phase diagram of HTS is the irreversibility line $[20,21]$ that separates the vortex glassy from the vortex liquid state. This line is determined by the temperature dependence of the irreversibility fields above that the flowing current forces the vortices to move. It means that energy dissipation appears and supercurrent vanishes. From this point of view, the irreversibility field of HTS plays a similar role to that of the upper critical field in classical superconductors. The temperature dependence of the irreversibility fields can be described by the following relation $[20,21]$ :

$H_{i r r}=H_{i r r 0}\left(1-\frac{T}{T_{c 0}}\right)^{n}$

where $H_{i r r}$ is the irreversibility field at $0 \mathrm{~K}, T_{\mathrm{c} 0}$ is the zero critical temperature at zero magnetic field and the exponent $n$ which is theoretically $3 / 2$, can vary in the wide range and it depends on the vortex properties. One can assume [16] that the temperature at which the whole sample stayed superconducting (resistance equal to zero) at the given magnetic field separates the reversibility from the irreversibility region. The data $H_{i r r}(T)$ were extracted from the magnetoresistance measurements and they are shown in Fig. 3 . The experimental data of $H_{i r r}(T)$ were successfully fitted

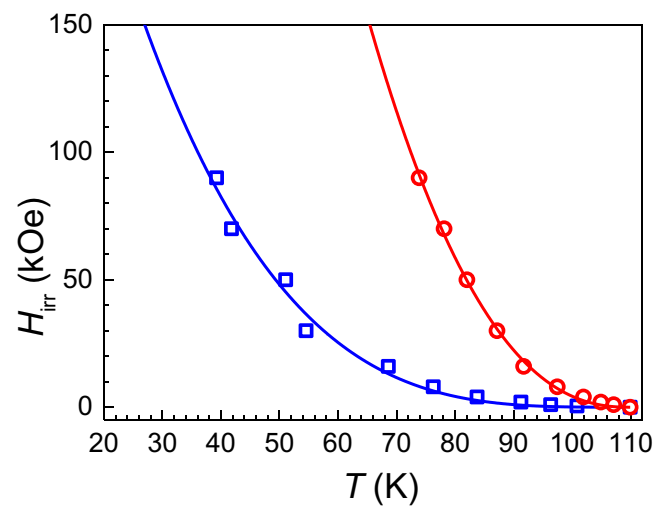

Fig. 3 Irreversibility fields as a function of temperature for the perpendicular (open squares) and the parallel (open circles) orientation of the applied magnetic field with respect to the surface of the tape. Solid lines are the fits to (2) using (2) with the two fit parameters: $n$ and $H_{i r r}$. The zerofield critical temperature $T_{\mathrm{c} 0}=109.9 \mathrm{~K}$ was taken from the experiment (solid lines in Fig. 3). The fit procedure delivers the following fit parameter values: $n=2.37 \pm 0.07$ and $H_{\text {irr }}=1280 \pm 110 \mathrm{kOe}$ for the parallel direction and $n=3.5 \pm 0.3$ and $H_{i r r}=400 \pm 60 \mathrm{kOe}$ for the perpendicular direction. Such high values of the exponents in (2) confirm that the pining forces of this tape are really strong. The irreversibility fields show distinct anisotropy and, at the liquid nitrogen temperature, they are as follows: $H_{i r r}=72.8 \mathrm{kOe}$ and $H_{i r r}=5.5 \mathrm{kOe}$ for the parallel and perpendicular directions, respectively. The anisotropy ratio can be written as follows [22]:

$\gamma=\frac{\lambda_{c}}{\lambda_{a b}}=\frac{H_{c 2 \mathrm{II} a b}}{H_{c 2 \mathrm{II} c}}=\frac{H_{i r r \mathrm{II} a b}}{H_{\mathrm{irrII} c}}$,

where the upper critical fields parallel to the $a b$ plane and to the $c$-axis, respectively, were replaced by the irreversibility fields suitable for both directions. For this tape, the anisotropy ratio amounts to $\gamma=13$ at $77 \mathrm{~K}$. One can say that if the magnetic field is perpendicular to the surface of the tape, it influences on the superconducting properties stronger than in the parallel direction. Similar dependencies were found for the critical current densities $J_{\mathrm{c}}(H)$ [23]. These behaviours can be explained assuming that the tape consists of the oriented grains that have $\mathrm{CuO}_{2}$ planes parallel to the surface of the tape. It means that the $a b$ planes are parallel and the $c$-axis is perpendicular to the surface of the tape, respectively. One can say that in this aspect the tape properties reflect with the two-dimensional nature of BSCCO superconductors.

\section{Conclusion}

The superconducting bismuth-based tape possesses good superconducting parameters: a high critical temperature $T_{\mathrm{c} 50 \%}=110.3 \mathrm{~K}$, a small width of the superconducting transition $\Delta T_{0}=0.5 \mathrm{~K}$, and a high critical current density $J_{\mathrm{c}}=1.710^{4} \mathrm{~A} / \mathrm{cm}^{-2}$ at $77 \mathrm{~K}$ in the own magnetic field. The magnetic field dependence of the width of superconducting transition and the temperature dependence of the irreversibility fields reveal an anisotropy that depends on the applied magnetic field orientations with respect to the surface of the tape. The width of superconducting transition versus magnetic field was fitted using (1) with the following fitted parameters: $m=0.41$ and $C=8.4$ for the perpendicular direction and $m=0.51$ and $C=2.04$ for the parallel direction. The critical exponents of these fits are generally smaller than the theoretical $m=2 / 3$ in (1). The irreversibility fields versus temperature were fitted using (2) with the following fitted parameters: $n=2.37$ and $H_{i r r}=1280 \mathrm{kOe}$ for the parallel direction and $n=3.5$ 
and $H_{i r r}=400 \mathrm{kOe}$ for perpendicular direction. The values of the exponents of (1) as well as (2) confirm that the pinning forces of this tape are really strong. Basing on the fit parameters of (2), the irreversibility fields at the liquid nitrogen temperature were calculated and they are as follows: $H_{i r r}=72.8 \mathrm{kOe}$ and $H_{i r r}=5.5 \mathrm{kOe}$ for the parallel and perpendicular directions, respectively. Using (3), the anisotropy ratio of the irreversibility fields' parallel and perpendicular orientations, respectively, of this tape was estimated and it amounts to $\gamma=13$ at $77 \mathrm{~K}$. A conclusion that the bismuth-based superconducting tape contains oriented grains that have $\mathrm{CuO}_{2}$ planes parallel to the surface of the tape could be taken into consideration basing on the results of this study.

Acknowledgments This work was supported by the Polish Ministry of Science and Higher Education and its grants for Scientific Research. The authors thank prof. H. Figiel and dr B. Proniewski for providing samples.

Open Access This article is distributed under the terms of the Creative Commons Attribution 4.0 International License (http:// creativecommons.org/licenses/by/4.0/), which permits unrestricted use, distribution, and reproduction in any medium, provided you give appropriate credit to the original author(s) and the source, provide a link to the Creative Commons license, and indicate if changes were made.

\section{References}

1. der Brake, H.J.M., Buchholz, F., Burnell, G., Claeson, T., Crete, D., Febvre, P., Gerritsma, G.J., Hilgenkamp, H., Humphreys, R., Ivanov, Z., Jutzi, W., Khabipov, M.I., Mannhart, J., Meyer, G.H., Niemeyer, J., Ravex, A., Rogalla, H., Russo, M., Satchell, J., Siegel, M., Topfer, M., Uhlmann, F.H., Villegier, J.-C., Wikborg, E., Woinkler, D., Zorin, A.B.: Physica C 439, 1 (2006)

2. Ayai, N., Ueyama, M., Kato, T., Kobayashi, S., Mikumo, T., Kaneko, T., Hikata, T., Hayashi, K., Takei, H.: Advances in Superconductivity XII, p. 631. Springer-Verlag, Tokyo (2000)
3. Nagasaki, Y., Nakamura, T., Funaki, I., Ashida, Y., Yamakawa, H.: Physica C 492, 96 (2013)

4. See for instance: Azman, J., Abdullah, H., Abd-Shukor, R.: Adv. Condens. Matter Phys. 1, 233 and references therein (2014)

5. Sunwong, S., Higgins, J.S., Hampshire, D.P.: IEEE Trans. Appl. Superconduct. 21, 2840 and references therein (2011)

6. Grassot, G., Jeremie, A., Flukiger, R.: Supercond. Sci. Technol. 8, 827 and references therein (1995)

7. Chunlong, Z., Yuntao, S., Xiongyi, H., Chenglian, L., Khan, S.U., Weibin, X., Kun, L.: Physica C 508, 17 (2015)

8. Letovic, M., Krishnaraj, P., Eror, N.G., Balachandran, U.: Physica C 242, 246 (1995)

9. Masur, L., Parker, D., Tanner, M., Podtburg, E., Buczek, D., Scudiere, J., Caracino, P., Spreafico, S., Corsaro, P., Nassi, M.: IEEE Trans. Appl. Supercond. 11, 3256 (2001)

10. Ma, Z., Taber, R.C., Lombardo, L.W., Kapitulnik, A., Beasley, M.R., Merchant, P., Eom, C.B., Hou, S.Y., Phillips, J.M.: Phys. Rev. Lett. 71, 781 (1993)

11. See for instance: 2004 High temperature superconductivity, Berlin 1 , Ed. A.V. Narlikar, Springer-Verlag, 1

12. Woch, W.M., Zalecki, R., Kołodziejczyk, A., Sudra, H., Gritzner, G.: Supercond. Sci. Technol. 21, 085002 (2008)

13. Woch, W.M., Chrobak, M., Zalecki, R., Kołodziejczyk, A.: Acta Phys. Pol. A 126, 328 (2014)

14. Palstra, T.T.M., Batlogg, B., Schneemeyer, L.F., Waszczak, J.V.: Phys. Rev. Lett. 61, 1662 (1988)

15. Kwok, W.K., Welp, U., Crabtree, G.W., Vandervoort, K.G., Hulscher, R., Liu, J.Z.: Phys. Rev. Lett. 64, 966 (1990)

16. Woch, W.M., Zalecki, R., Chrobak, M., Kołodziejczyk, A., Gritzner, G.: Acta Phys. Pol. A 121, 789 (2012)

17. Woch, W.M., Zalecki, R., Kołodziejczyk, A., Heiml, O., Gritzner, G.: Physica C 434, 17 (2006)

18. Yeshurun, Y., Malozemoff, A.P.: Phys. Rev. Lett. 60, 2202 (1988)

19. Woch, W.M., Zalecki, R., Chrobak, M., Kołodziejczyk, A.: Acta Phys. Pol. A 127, 315 (2015)

20. Müller, K.A., Takashige, M., Bednorz, J.: Phys. Rev. Lett. 58, 1143 (1987)

21. de Almeida, J.R.L., Thouless, D.J.: J. Phys. A 11, 983 (1978)

22. Tinkham, M.: Introduction to Superconductivity. McGraw-Hill Inc, New York (1996)

23. Sunwong, P., Higgins, J.S., Hampshire, D.P.: IEEE Trans. Applied Supercond. 21, 2840 (2011) 